\title{
Validation of the Palliative Prognostic Index, Performance Status-Based Palliative Prognostic Index and Chinese Prognostic Scale in a home palliative care setting for patients with advanced cancer in China
}

Jun Zhou ${ }^{1+}$, Sitao $\mathrm{Xu}^{1+}$, Ziye Cao ${ }^{1}$, Jing Tang ${ }^{1}$, Xiang Fang ${ }^{1}$, Ling Qin ${ }^{1}$, Fangping Zhou ${ }^{2}$, Yuzhen $\mathrm{He}^{3,2}$, Xueren Zhong ${ }^{1}$, Mingcai $\mathrm{Hu}^{3}$, Yan Wang ${ }^{4}$, Fengjuan $\mathrm{Lu}^{5}$, Yongzheng Bao ${ }^{1}$, Xiangheng Dai ${ }^{6^{*}}$ and Qiang $\mathrm{Wu}^{{ }^{*}}$

\begin{abstract}
Background: The predictive value of the prognostic tool for patients with advanced cancer is uncertain in mainland China, especially in the home-based palliative care (HPC) setting. This study aimed to compare the accuracy of the Palliative Prognostic Index (PPI), the Performance Status-Based Palliative Prognostic Index (PS-PPI), and the Chinese Prognosis Scale (ChPS) for patients with advanced cancer in the HPC setting in mainland China.

Methods: Patients with advanced cancer admitted to the hospice center of Yuebei People's Hospital between January 2014 and December 2018 were retrospectively calculated the scores according to the three prognostic tools. The Kaplan-Meier method was used to compare survival times among different risk groups. Receiver operating characteristic curve analysis was used to assess the predictive value. The accuracy of 21-, 42- and 90-day survival was compared among the three prognostic tools.

Results: A total of 1863 patients were included. Survival time among the risk groups of all prognostic tools was significantly different from each other except for the PPI. The AUROC of the ChPS was significantly higher than that of the PPI and PS-PPI for 7-, 14, 21-, 42-, 90-, 120-, 150- and 180-day survival $(P<0.05)$. The AUROC of the PPI and PS-PPI were not significantly different from each other $(P>0.05)$.
\end{abstract}

Conclusions: The ChPS is more suitable than the PPI and PS-PPI for advanced cancer patients in the HPC setting. More researches are needed to verify the predictive value of the ChPS, PPI, and PS-PPI in the HPC setting in the future.

Keywords: Validation, Palliative prognostic index, Performance status-based palliative prognostic index, Chinese prognosis scale, Advanced Cancer, Home-based palliative care setting

\footnotetext{
*Correspondence: daixiangheng@163.com; sgwuqiang@sina.com

†Jun Zhou and Sitao Xu contributed equally to this work.

${ }^{6}$ Department of Spinal Surgery, Nanfang Hospital, Southern Medical University, Guangzhou, Guangdong, China

'Department of Spine Surgery, Yuebei People's Hospital Affiliated to Shantou University Medical College, Shaoguan, Guangdong, China

Full list of author information is available at the end of the article
}

(c) The Author(s). 2020 Open Access This article is licensed under a Creative Commons Attribution 4.0 International License, which permits use, sharing, adaptation, distribution and reproduction in any medium or format, as long as you give appropriate credit to the original author(s) and the source, provide a link to the Creative Commons licence, and indicate if changes were made. The images or other third party material in this article are included in the article's Creative Commons licence, unless indicated otherwise in a credit line to the material. If material is not included in the article's Creative Commons licence and your intended use is not permitted by statutory regulation or exceeds the permitted use, you will need to obtain permission directly from the copyright holder. To view a copy of this licence, visit http://creativecommons.org/licenses/by/4.0/. The Creative Commons Public Domain Dedication waiver (http://creativecommons.org/publicdomain/zero/1.0/) applies to the data made available in this article, unless otherwise stated in a credit line to the data. 


\section{Background}

It is one of the basic parts of palliative care to predict the survival of patients with advanced cancer accurately $[1,2]$. In the final stages of a cancer patient's life, predictions of survival will help the patient and family decide whether to continue treatment and help achieve patients' last wishes [1, 3-5]. Clinical prediction of survival tends to overestimate the actual survival time of advanced cancer patients because clinical prediction of survival is based on the experience of physicians [6-8]. A previous study revealed that predicting with the application of prognostic tools can provide the patient and family with more accurate prognostic information [9].

Prognostic tool plays an important role in palliative care, but the study about prognostic tools for advanced cancer patients is still in its infancy in mainland China $[10,11]$. An estimated 4.29 million new cancer cases and 2.81 million cancer deaths occurred in China in 2015 [12]. There have been more than 30 hospice centers sponsored by $\mathrm{Li} \mathrm{Ka}$ Shing Foundation in mainland China, which provide home-based palliative care services for around 16 thousand economically disadvantaged patients with advanced cancer living in urban and remote rural villages each year [13]. Prognostic tools are necessary for the home-based palliative care (HPC) setting because palliative care specialists are possible to provide more accurate prognostic information with these tools when communicating with the patient and family.

Several prognostic tools have been developed and validated, which are frequently applied to predict the survival of advanced cancer patients in the hospital palliative care setting [14-22]. However, a blood test is not always available for patients with advanced cancer, especially those in the HPC setting [23]. Comparing with other validated prognostic tools, the Palliative Prognostic Index (PPI) consisting of five independently predictive variables may be more suitable for advanced cancer patients in the HPC setting, for which does not require any invasive procedure [23-25]. Previous studies reported that the PPI had a low sensitivity for patients with advanced cancer in the HPC setting [25-30]. However, there is no study to support the validity of the PPI in the HPC setting in mainland China $[24,25]$. Recently, Takeshi et al. [31] reported the development of the Performance Status-Based Palliative Prognostic Index (PS-PPI), a brief version of the PPI, in which the performance status was based on the Eastern Cooperative Oncology Group (ECOG) PS instead of the Palliative Performance Scale (PPS). The sensitivity of the PS-PPI for advanced cancer patients was higher than that of the PPI. Further validations for the PS-PPI in other palliative care settings are needed. Besides, the Chinese Prognosis Scale (ChPS), the first prognostic scale in mainland China, was developed based on patients in the HPC setting sponsored by
Li Ka Shing Foundation in Shanghai, China by Zhou et al. [10]. However, the ChPS has not been fully validated by other research teams.

It is still uncertain whether the three prognostic tools are suitable for patients with advanced cancer in the HPC setting in mainland China. Therefore, the purpose of the present study was to validate and compare the predictive value of the PPI, PS-PPI and ChPS for patients with advanced cancer in the HPC setting in mainland China.

\section{Methods}

This retrospective observational study was conducted on patients consecutively admitted to the hospice center of Yuebei People's Hospital sponsored by the Li Ka Shing Foundation between January 2014 and December 2018. In this study, patients who satisfied the following criteria were included: (1) at least 18 years old; (2) must be diagnosed with locally extensive or metastatic advanced cancer in a "high-level hospital", including hematological neoplasm; (3) agree to participate in palliative home care service. Patients who terminated the services halfway or data missing were excluded. Patients' demographic information (age, gender, site of primary cancer and metastatic disease and survival time) and clinical characteristics (performance status, symptoms, and signs) were assessed and recorded by a palliative care team, consisting of 2 physicians, 2 specialist nurses, and 1 social worker at the first consultation. All patients were followed up by home visits or phone calls on a regular basis until the end of the service when patients passed away. The calculation of the survival time was from the date of the first assessment until the date of death. The study was approved by the ethical review board of Yuebei People's Hospital Affiliated to Shantou University Medical College (KY-2019-024) and was performed according to the ethical standards laid down in the 1964 Declaration of Helsinki and its later amendments or comparable ethical standards. Written informed consent was obtained from all individual or guardian participants.

\section{Chinese prognosis scale}

The ChPS was initially developed by Zhou et al. in 2009 to predict the survival of advanced cancer patients in the HPC setting [10]. The ChPS score was calculated by summing the scores of ten prognostic factors: weight loss, nausea, dysphagia, dyspnea, edema, cachexia, dehydration, gender, KPS (Karnofsky Performance Status) scores, and QOL (Quality of Life) scores [32]. [33] The range of ChPS scores is from 0 (no altered variables) to 124 (maximal altered variables). Patients were classified into 2 risk groups according to the original article: group A included patients with ChPS scores $\leq 28$ and group B with ChPS scores $>28$. A ChPS score of more than 28 
predicts survival of less than 90 days, and a score of less than or equal to 28 predicts survival of 90 to 180 days.

\section{Palliative prognostic index}

The PPI was initially developed by Morita et al. in 1999 to predict the survival of advanced cancer patients in palliative care units [24]. The PPI score was calculated by summing the scores of five independently predictive variables: PPS scores, oral intake, edema, dyspnea at rest, and delirium [21]. The PPS scores in the present study were transferred from the KPS scores. KPS scores of 10100 corresponded to PPS scores of 10-100, respectively [34]. The range of PPI scores is from 0 to 15. Patients were classified into 3 risk groups according to the original article: group A (0.0-4.0), group B (4.1-6.0), and group C (6.1-15.0). Patients with a PPI score of more than 6 survive less than 21 days, and with a score of more than 4 survive less than 42 days.

\section{Performance status-based palliative prognostic index}

Using the Eastern Cooperative Oncology Group (ECOG) PS to assess advanced cancer patients' performance status instead of the Palliative Performance Scale (PPS), the PS-PPI was recently developed by Takeshi et al. in 2016 to predict the survival of advanced cancer patients [31]. The PS-PPI score was calculated by summing the scores of the ECOG PS scores, oral intake, delirium, dyspnea at rest, and edema [35]. The ECOG PS scores in the present study were transferred from the KPS scores. KPS scores of 100, 90-80, 70-60, 50-40, and 30-10 corresponded to ECOG PS scores of $0,1,2,3$, and 4, respectively [34]. The range of PS-PPI scores is from 0 to 15 . Patients were classified into 3 risk groups according to the original article: group A (0.0-4.0), group B (4.1-6.0), and group $C(6.1-15.0)$. A PS-PPI score of more than 6 predicts survival of less than 21 days, and a score of more than 4 predicts survival of less than 42 days.

\section{Statistical analysis}

General characteristics of patients and variables of the three prognostic tools were summarized and analyzed. Survival curves were estimated using the Kaplan-Meier method, and the log-rank test was used to compare survival times among each risk group of these three prognostic tools. The area under the receiver operating characteristic curve (AUROC) was calculated to determine the accuracy of the three prognostic tools for predicting survival time within $7,14,21,30,42,60,90,120$, 150 , and 180 days. The AUROC of the PPI, PS-PPI and ChPS were compared respectively based on the DeLong method [36]. Sensitivity, specificity, positive predictive value (PPV), negative predictive value (NPV), and overall accuracy $(\mathrm{OA})$ were calculated for prediction of 21-day, 42-day, and 90-day survival using the best cutoff score, which was decided by Youden index. In all analyses, $P<$ 0.05 was defined as significance. Survival time was presented as median (95\% CI, confidence intervals), and continuous data was presented as mean (SD, standard deviation). MedCalc version 18.2.1 (MedCalc Software, Ostend, Belgium) was used to compare the AUROC among the three prognostic tools. Additional statistical analyses were carried out with IBM SPSS Statistics Version 22.0.0.0 (SPSS Inc., Chicago, IL) and GraphPad Prism Version 8.0.2 (GraphPad Software, San Diego, CA).

\section{Results}

\section{General characteristics of patients}

A total of 1863 patients were included in the study. Table 1 shows background information of patients in detail. The mean age of patients was $61.5 \pm 12.64$ years, and males accounted for $62.6 \%$ of the sample. The most prevalent primary cancer sites of the patients were as follows: lung (31.6\%), liver (14.9\%), and colon/rectum/ small intestine (12.1\%). The median survival time of patients was 52 days.

\section{Survival analysis of the three prognostic tools}

Particular variables of the three prognostic tools are shown in Table 2. Risk groups of the three prognostic tools and median survival time of patients are shown in Table 3. The values for median survival and relative 95\% $\mathrm{CI}$ and distribution for the three risk groups of the PPI were 69 days $(95 \% \mathrm{CI} 63-74$ days) in group A (0.0-4.0; $73.1 \%), 31$ days (95\%CI $26-37$ days) in group B (4.1-6.0; $15.8 \%), 22$ days $(95 \% \mathrm{CI} 17-27$ days) in group $\mathrm{C}(6.1-$ 15.0; $11.1 \%$; the values for median survival and relative 95\% CI and distribution for the three risk groups of the PS-PPI were 105 days (95\%CI 93-116 days) in group A $(0.0-4.0 ; 21.8 \%), 55$ days $(95 \%$ CI $50-60$ days) in group B (4.1-6.0; 51.0\%), 27 days (95\%CI $24-30$ days) in group $\mathrm{C}$ (6.1-15.0; 27.2\%); the values for median survival and relative $95 \% \mathrm{CI}$ and distribution for the two risk groups of the ChPS were 103 days (95\%CI 93-114 days) in group A (0.0-28.0; $21.5 \%), 44$ days (95\% CI 41-47 days) in group B (28.1-124.0; 78.5\%). The survival times of the risk groups of the PS-PPI and ChPS were significantly different from each other $(P<0.001)$. Nevertheless, the survival times of the risk groups of the PPI were not significantly different from each other $(P=0.089)$. KaplanMeier survival curves are shown in Fig. 1.

\section{Accuracy of the three prognostic tools}

Receiver operating characteristic curves for 21-, 42-, 90and 180-day survival of the three prognostic tools were compared (Fig. 2 a, b, c). The AUROC values for 7-, 14-, 21-, 42-, 60-, 90-, 120-, 150- and 180-day survival were $0.776,0.733,0.709,0.693,0.688,0.67,0.668,0.658,0.661$ and 0.659 , respectively for the PPI; were $0.773,0.729$, 
Table 1 General Characteristics of Patients $(N=1863)$

\begin{tabular}{|c|c|}
\hline Characteristics & Mean \pm SD or $N(\%)$ \\
\hline Age, years & $61.5 \pm 12.64$ \\
\hline \multicolumn{2}{|l|}{ Gender } \\
\hline Male & $1166(62.6)$ \\
\hline Female & $697(37.4)$ \\
\hline \multicolumn{2}{|l|}{ Site of primary cancer } \\
\hline Lung & $590(31.7)$ \\
\hline Esophagus/stomach & $171(9.2)$ \\
\hline Colon/rectum/small intestine & $226(12.1)$ \\
\hline Liver & $277(14.9)$ \\
\hline Pancreas & $60(3.2)$ \\
\hline Biliary system & $36(1.9)$ \\
\hline Breast & $84(4.5)$ \\
\hline Kidney/renal pelvis/ureter/bladder/prostate & $56(3)$ \\
\hline Ovary/uterus & $103(5.5)$ \\
\hline Head and neck (incl thyroid) & $141(7.6)$ \\
\hline Blood (leukaemia/myeloma/lymphoma) & $27(1.4)$ \\
\hline Central nervous system & $10(0.5)$ \\
\hline Soft tissue (Sarcoma) & $15(0.8)$ \\
\hline Unknown & $29(1.6)$ \\
\hline Other & $45(2.4)$ \\
\hline \multicolumn{2}{|l|}{ Metastatic site } \\
\hline Any site & $1590(85.3)$ \\
\hline Liver & $438(23.5)$ \\
\hline Lung & $382(20.5)$ \\
\hline Bone & $512(27.5)$ \\
\hline Central nervous system & $133(7.1)$ \\
\hline Survival time (day) ${ }^{a}$ & $52(49.0-56.0)$ \\
\hline$<21$ & $385(20.7)$ \\
\hline$<42$ & $773(41.5)$ \\
\hline$<90$ & $1225(65.8)$ \\
\hline$<180$ & $1552(83.3)$ \\
\hline
\end{tabular}

Abbreviation: $S D$ standard deviation

${ }^{a}$ Data were expressed as median (95\% confidence intervals)

0.707, 0.692, 0.687, 0.67, 0.667, 0.658, 0.659 and 0.658, respectively for the PS-PPI; were $0.815,0.77,0.734,0.718$, $0.709,0.686,0.697,0.687,0.692$ and 0.683 , respectively for the ChPS. The AUROC of the ChPS was significantly higher than that of the PPI and PS-PPI for 7-, 14, 21-, 42-, 90-, 120-, 150- and 180-day survival $(P<0.05)$. The AUROCs of the PPI and PS-PPI were not significantly different from each other $(P>0.05)$ (Fig. $2 \mathrm{~d})$. The best cutoff scores for 21-day survival were 4.5 for the PPI, 6 for the PS-PPI, and 39 for the ChPS. The best cutoff scores for 42-day survival were 4 for the PPI, 6 for the PS-PPI, and 35 for the ChPS. The best cutoff scores for 90-day survival were 4 for the PPI, 4.5 for the PS-PPI, and 38 for the ChPS.
Table 2 Variables of the Three Prognostic Tools

\begin{tabular}{|c|c|c|c|}
\hline \multicolumn{2}{|l|}{ PPI and PS-PPI } & \multicolumn{2}{|l|}{ ChPS } \\
\hline Variables & $N(\%)$ & Variables & $N(\%)$ \\
\hline PPS & & Weight loss & \\
\hline $10-20$ & $2(0.1)$ & No & $22(1.2)$ \\
\hline $30-50$ & $1382(74.2)$ & Yes & $1841(98.8)$ \\
\hline$\geq 60$ & $479(25.7)$ & Nausea & \\
\hline Oral intake & & No & $1347(72.3)$ \\
\hline Normal & $15(0.8)$ & Yes & $516(27.7)$ \\
\hline Moderately reduced & $1679(90.1)$ & Dysphagia & \\
\hline Severely reduced & $169(9.1)$ & No & $1775(95.3)$ \\
\hline Edema & & Yes & $88(4.7)$ \\
\hline No & $1582(84.9)$ & Dyspnea & \\
\hline Yes & $281(15.1)$ & No & $1683(90.3)$ \\
\hline Dyspnea at rest & & Yes & $180(9.7)$ \\
\hline No & $1683(90.3)$ & Edema & \\
\hline Yes & $180(9.7)$ & No & $1582(84.9)$ \\
\hline Delirium & & Yes & $281(15.1)$ \\
\hline No & $1825(98.0)$ & Gender & \\
\hline Yes & $38(2.0)$ & Male & $1166(62.6)$ \\
\hline ECOG PS & & Female & $697(37.4)$ \\
\hline $0-1$ & $2(0.1)$ & Cachexia & \\
\hline 2 & $477(25.6)$ & No & $1745(93.7)$ \\
\hline \multirow[t]{13}{*}{$3-4$} & $1384(74.3)$ & Yes & $118(6.3)$ \\
\hline & & Dehydration & \\
\hline & & No & $1772(95.1)$ \\
\hline & & Yes & $91(4.9)$ \\
\hline & & $\mathrm{QOL}$ & \\
\hline & & $>40$ & $37(2.0)$ \\
\hline & & $31-40$ & $1224(65.7)$ \\
\hline & & $0-30$ & $602(32.3)$ \\
\hline & & KPS & \\
\hline & & $>70$ & $2(0.1)$ \\
\hline & & 70 & $30(1.6)$ \\
\hline & & 60 & $447(24.0)$ \\
\hline & & $\leq 50$ & $1384(74.3)$ \\
\hline
\end{tabular}

Abbreviations: PPI Palliative Prognostic Index, PS-PPI Performance Status-Based Palliative Prognostic Index, ChPS Chinese Prognostic Scale, PPS Palliative Performance Scale, ECOG PS Eastern Cooperative Oncology Group

Performance Status, KPS Karnofsky Performance Status, QOL Quality of Life

Besides, the sensitivity, specificity, PPV, NPV values and OA of the three prognostic tools are showed in Table 4.

\section{Discussion}

In the present study, the three prognostic tools were validated and compared simultaneously for predicting the survival of patients with advanced cancer in mainland China, especially for patients who received home-based 
Table 3 Median Survival Time of the Three Prognostic Tools

\begin{tabular}{|c|c|c|c|c|c|c|c|c|}
\hline \multicolumn{3}{|l|}{ PPI } & \multicolumn{3}{|l|}{ PS-PPI } & \multicolumn{3}{|l|}{ ChPS } \\
\hline $\begin{array}{l}\text { Risk groups } \\
\text { (total scores) }\end{array}$ & $\begin{array}{l}\text { Number of } \\
\text { patients (\%) }\end{array}$ & $\begin{array}{l}\text { Median survival } \\
\text { (days) }(95 \% \mathrm{Cl})\end{array}$ & $\begin{array}{l}\text { Risk groups } \\
\text { (total scores) }\end{array}$ & $\begin{array}{l}\text { Number of } \\
\text { patients (\%) }\end{array}$ & $\begin{array}{l}\text { Median survival } \\
\text { (days) }(95 \% \mathrm{Cl})\end{array}$ & $\begin{array}{l}\text { Risk groups } \\
\text { (total scores) }\end{array}$ & $\begin{array}{l}\text { Number of } \\
\text { patients (\%) }\end{array}$ & $\begin{array}{l}\text { Median survival } \\
\text { (days) }(95 \% \mathrm{Cl})\end{array}$ \\
\hline$\overline{A(0.0-4.0)}$ & $1361(73.1)$ & $69(63-74)$ & $A(0.0-4.0)$ & $407(21.8)$ & $105(93-116)$ & $\mathrm{A}(0.0-28.0)$ & $401(21.5)$ & $103(93-114)$ \\
\hline B (4.1-6.0) & 295 (15.8) & $31(26-37)$ & $B(4.1-6.0)$ & $950(51.0)$ & $55(50-60)$ & B (28.1-124.0) & $1462(78.5)$ & $44(41-47)$ \\
\hline$C(6.1-15.0)$ & $207(11.1)$ & $22(17-27)$ & $C(6.1-15.0)$ & $506(27.2)$ & $27(24-30)$ & & & \\
\hline
\end{tabular}

Abbreviations Cl, confidence intervals; PPI Palliative Prognostic Index; PS-PPI Performance Status-Based Palliative Prognostic Index; ChPS Chinese Prognostic Scale

palliative care services. Our results indicate that the ChPS is more suitable for advanced cancer patients in the HPC setting than the PPI and PS-PPI.

The ChPS was initially developed for predicting the survival of advanced cancer patients in the HPC setting in China [10]. Group B (total scores 28.1-124.0) in ChPS exhibited a significantly shorter survival time than group A (total scores $0.0-28.0$ ) in the present study, which is consistent with the results of Zhou et al. [10] The overall accuracy for predicting the survival of less than 90 days with a ChPS score of 28 was $68.3 \%$, which is similar to the outcome from the original article that the overall accuracy of the testing set was $65.4 \%$ [10]. In the present study, the AUROC of the ChPS was significantly higher than that of the PPI and PS-PPI for 7-, 14, 21-, 42-, 90-, $120-, 150$ - and 180-day survival, and the sensitivity and PPV of the ChPS for predicting 90-day survival were 86.1 and $71.5 \%$, respectively. However, the ChPS had low specificity and NPV in the present study. Low NPV indicated that there were some patients with ChPS scores (0.0-28.0) who lived less than 90 days. In practice, false-positive predictions are more critical than falsenegative predictions because the predicted survival time being longer than the actual survival time may induce the problem in communication between clinicians and patients or their family for decision making [37, 38]. Based on this view, the ChPS could be utilized as a screening tool for prognostication because of its high sensitivity and PPV, which is a prerequisite for a useful screening tool. Besides, the classification of primary cancer was included in some scoring systems in previous studies [39-41]. Patients with different types of cancer may have individual survival time because primary cancer is considered a significant factor related to survival time. In addition, previous studies reported that the specific site of metastasis was associated with survival time [42-44]. Yin et al. [42] reported that liver metastasis was regarded as an independent predictor related to poor prognosis for patients with cervical cancer. Another study reported that metastatic renal cell carcinoma patients with isolated liver metastases seemed to have worse outcomes [43]. However, the primary site of the tumor and the specific site of metastasis were not considered in the ChPS. Further studies are needed to confirm that the accuracy of the ChPS could be improved by including factors relative to the primary site of the tumor and the particular site of metastasis.

In a retrospective study, Hamano et al. [27] suggested that the PPI might not be suitable as a screening tool for poor prognosis patients with relatively good performance
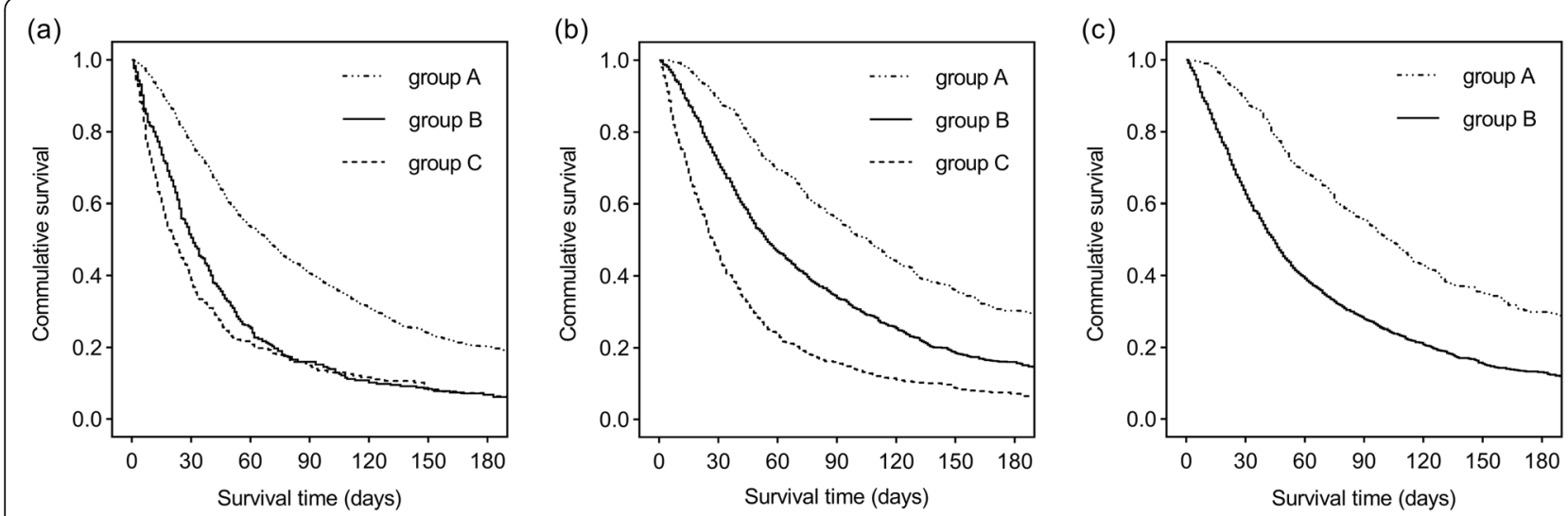

Fig. 1 Kaplan-Meier survival curves of the risk groups categorized by the three prognostic tools. a, Palliative Prognostic Index: group A (0.0-4.0), group B (4.1-6.0), and group C (6.1-15.0). b, Performance Status-Based Palliative Prognostic Index: group A (0.0-4.0), group B (4.1-6.0), and group C (6.1-15.0). c, Chinese Prognostic Scale: group A (0.0-28.0) and group B (28.1-124.0). Log-rank tests were all significant for the 3 prognostic tools $(P<0.001)$, except survival time between group $B$ and group $C(P=0.089)$ in Palliative Prognostic Index. 
Survival time $<21$ days

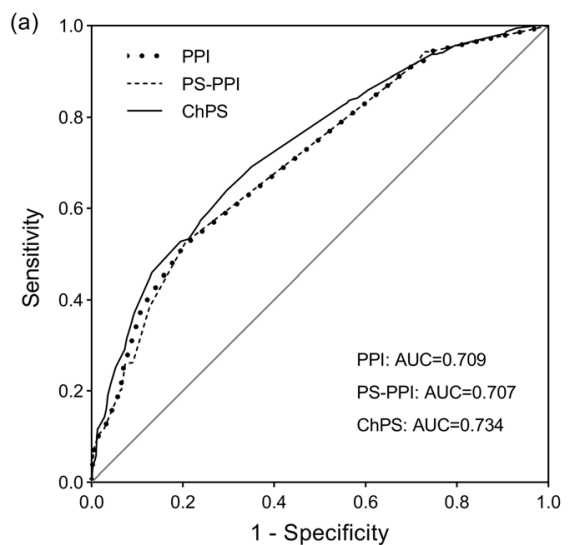

Survival time $<90$ days

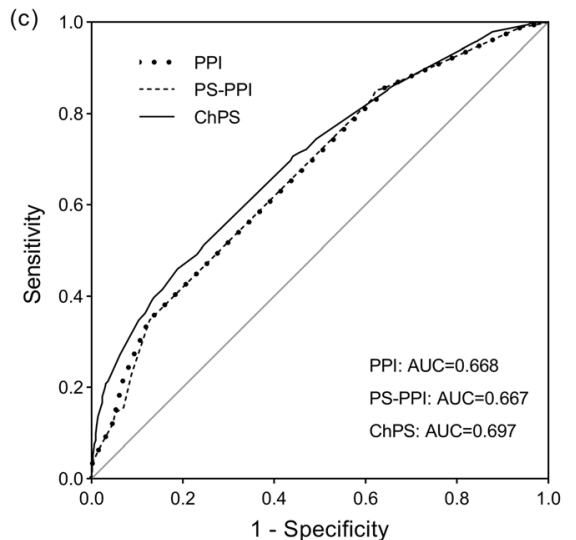

Survival time $<42$ days

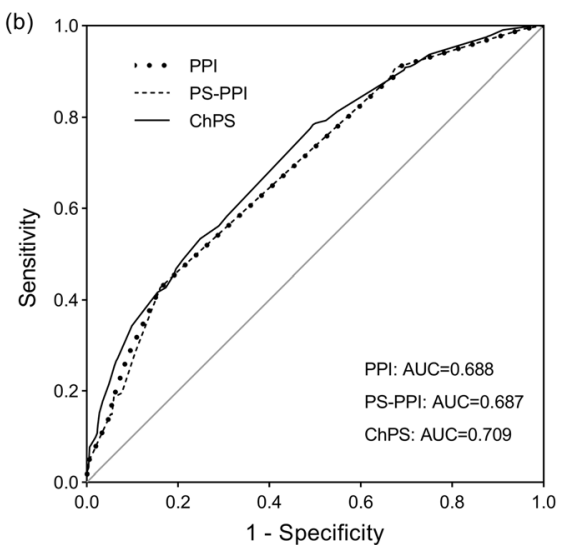

(d)

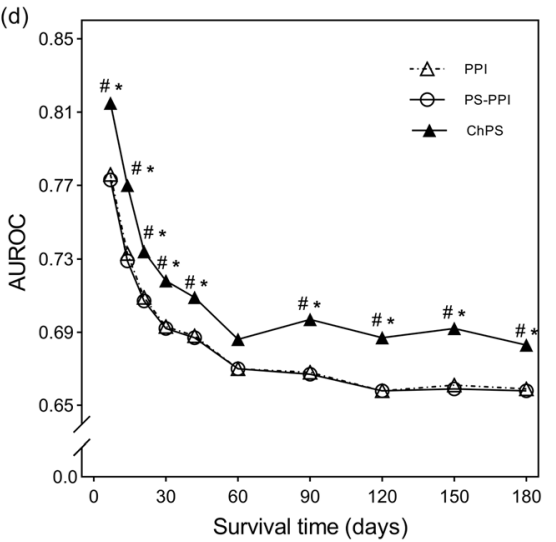

Fig. 2 Comparison of the AUROC for 21-day, 42-day, and 90-day survival among the three prognostic tools. a, The AUROC values for 21-day survival were 0.709 for the PPI, 0.707 for the PS-PPI, and 0.734 for the ChPS. The best cutoff scores for 21-day survival were 4.5 for the PPI, 6 for the PS-PPI, and 39 for the ChPS. b, The AUROC values for 42-day survival were 0.688 for the PPI, 0.687 for the PS-PPI, and 0.709 for the ChPS. The best cutoff scores for 42-day survival were 4 for the PPI, 6 for the PS-PPI, and 35 for the ChPS. c, The AUROC values for 90 -day survival were 0.668 for the PPI, 0.667 for the PS-PPI, and 0.697 for the ChPS. The best cutoff scores for 90 -day survival were 4 for the PPI, 4.5 for the PS-PPI, and 38 for the ChPS. d, Comparison of the AUROC values for 7-, 14-, 21-, 30-, 42-, 60-, 90-, 120-, 150- and 180-day survival among the three prognostic tools. \# *AUROC are significantly higher in the ChPS than both PPI and PS-PPI. * $P<0.05$, ChPS versus PPI; \# $P<0.05$, ChPS versus PS-PPI. Abbreviation: AUROC, Area Under the Receiver Operating Characteristic Curve; PPI, Palliative Prognostic Index; PS-PPI, Performance Status-Based Palliative Prognostic Index; ChPS, Chinese Prognostic ScaleAll tables could be placed at the end of Results during production.

status in the home care setting considering its low sensitivity, but might be suitable for predicting survival longer than 21 days because of its high specificity. A further prospective study supported this finding [29]. A similar outcome was presented in our study that the sensitivity for 21-day and 42-day survival of the PPI was lower than that of the original article when the cutoff score was set at 6.0 and 4.0, respectively, and the PPI had a high specificity with the same cutoff scores. The discrepancy of the sensitivity may be attributed to differences between the patients. The low sensitivity in the HPC setting could be interpreted by the lowest prevalence for PPI $>6$ (11.1\%), which indicated that patients with advanced cancer in the HPC setting may be in a better general condition and have fewer complications $[10,45]$. Characteristics related to the survival of patients were different for various studies $[29,46]$. The median survival time of the patients in the present study was 52 days, whereas Morita et al. [24] reported 27 days, and Maltoni et al. [30] reported 22 days in the hospice setting. In addition, the prevalence of severely reduced oral intake, edema, dyspnea at rest, and delirium was lower than those in the original article. Some scholars hold the view that a one-shot PPI assessment might not be accurate enough as a prognostic tool because patients' clinical features changed dynamically during the end-of-life trajectory [47-49]. Arai et al. [49] reported a retrospective cohort study that reassessment of the PPI was necessary because of the change in the PPI as an important and independent factor associated with the survival of advanced cancer patients. Another previous study reported by Kao et al. [48] showed that the 
Table 4 Accuracy of the Three Prognostic Tools

\begin{tabular}{|c|c|c|c|c|c|c|}
\hline & Cutoff & Sensitivity $(\%, 95 \mathrm{Cl})$ & Specificity $(\%, 95 \mathrm{Cl})$ & PPV $(\%, 95 \mathrm{Cl})$ & NPV $(\%, 95 \mathrm{Cl})$ & $\mathrm{OA}(\%, 95 \mathrm{Cl})$ \\
\hline \multicolumn{7}{|l|}{21 days } \\
\hline \multirow[t]{3}{*}{ PPI } & $4.5^{\mathrm{a}}$ & $52.0(46.8-57.0)$ & $79.6(77.5-81.7)$ & $39.9(36.6-43.3)$ & $86.4(85.1-87.6)$ & $73.9(70.1-77.9)$ \\
\hline & 5 & $38.2(33.3-43.2)$ & $89.0(87.3-90.6)$ & $47.6(42.8-52.4)$ & $84.7(83.6-85.7)$ & $78.5(74.5-82.6)$ \\
\hline & 6 & $26.2(21.9-30.9)$ & $92.8(91.4-94.1)$ & $48.8(42.6-55.0)$ & $82.9(82.0-83.7)$ & $79.0(75.0-83.2)$ \\
\hline \multirow[t]{3}{*}{ PS-PPI } & $6^{a}$ & $52.5(47.3-57.6)$ & 79.4 (77.3-81.5) & $40.0(36.7-43.3)$ & $86.5(85.2-87.7)$ & $73.8(70.0-77.9)$ \\
\hline & 6.5 & $38.4(33.6-43.5)$ & $87.3(85.5-89.9)$ & $44.0(39.6-48.6)$ & $84.5(83.4-85.5)$ & $77.2(73.3-81.3)$ \\
\hline & 7 & $26.2(21.9-30.9)$ & 90.9 (89.4-92.3) & $43.0(37.4-48.7)$ & $82.6(81.6-83.4)$ & 77.5 (73.6-81.6) \\
\hline \multirow[t]{3}{*}{ ChPS } & 38 & $67.3(62.3-71.9)$ & $66.9(64.4-69.2)$ & $34.6(32.3-36.9)$ & $88.7(87.1-90.1)$ & $67.0(63.3-70.8)$ \\
\hline & $39^{a}$ & $63.9(58.9-68.7)$ & $70.4(68.0-72.8)$ & $36.0(33.6-38.6)$ & $88.2(86.7-89.6)$ & $69.1(65.4-73.0)$ \\
\hline & 40 & $59.2(54.1-64.2)$ & $74.4(72.1-76.6)$ & $37.6(34.8-40.4)$ & 87.5 (86.1-88.8) & $71.3(67.5-75.2)$ \\
\hline \multicolumn{7}{|l|}{42 days } \\
\hline \multirow[t]{3}{*}{ PPI } & 3.5 & 88.1 (85.6-90.3) & $33.9(31.1-36.8)$ & $48.6(47.4-49.9)$ & $80.1(76.5-83.2)$ & $56.4(53.1-59.9)$ \\
\hline & $4^{a}$ & $42.4(38.9-46.0)$ & $84.0(81.7-86.2)$ & $65.3(61.7-68.8)$ & $67.3(65.8-68.7)$ & $66.7(63.1-70.5)$ \\
\hline & 4.5 & $42.3(38.8-45.9)$ & $84.0(81.7-86.2)$ & $65.3(61.6-68.8)$ & $67.3(65.8-68.7)$ & $66.7(63.1-70.5)$ \\
\hline \multirow[t]{3}{*}{ PS-PPI } & 4 & $91.2(89.0-93.1)$ & $31.1(28.4-34.0)$ & $48.5(45.9-51.1)$ & $83.3(79.2-86.7)$ & $56.0(52.7-59.5)$ \\
\hline & 5 & 88.6 (86.2-90.8) & $33.7(30.9-36.6)$ & $48.7(47.4-49.9)$ & $80.7(77.1-83.8)$ & $56.5(53.1-60.0)$ \\
\hline & $6^{a}$ & $42.7(39.2-46.3)$ & $83.9(81.5-86.0)$ & $65.2(61.6-68.7)$ & $67.4(65.9-68.8)$ & $66.8(63.2-70.7)$ \\
\hline \multirow[t]{3}{*}{ ChPS } & 34 & $78.8(75.7-81.6)$ & $49.9(46.9-52.9)$ & $52.7(51.0-54.5)$ & $76.8(74.1-79.4)$ & $61.9(58.4-65.6)$ \\
\hline & $35^{a}$ & $78.5(75.5-81.4)$ & $50.5(47.4-53.5)$ & $52.9(51.1-54.6)$ & $76.7(74.0-79.2)$ & $62.1(58.6-65.8)$ \\
\hline & 36 & $77.6(74.5-80.5)$ & $51.1(48.1-54.1)$ & $53.0(51.2-54.7)$ & 76.3 (73.6-78.8) & $62.1(58.6-65.8)$ \\
\hline \multicolumn{7}{|l|}{90 days } \\
\hline \multirow[t]{3}{*}{ PPI } & 3.5 & $82.5(80.2-84.5)$ & $38.7(34.9-42.6)$ & 72.1 (70.7-73.4) & $53.5(49.6-57.3)$ & $67.5(63.9-71.4)$ \\
\hline & $4^{a}$ & $34.5(31.9-37.3)$ & $87.6(84.8-90.1)$ & $84.3(81.1-87.0)$ & $41.1(39.9-42.3)$ & $52.7(49.5-56.1)$ \\
\hline & 4.5 & $34.5(31.8-37.2)$ & $87.6(84.8-90.1)$ & $84.2(81.1-86.9)$ & $41.0(39.8-42.3)$ & $52.7(49.5-56.1)$ \\
\hline \multirow[t]{3}{*}{ PS-PPI } & 4 & $85.6(83.5-87.5)$ & 36.1 (32.3-39.9) & $72.0(70.7-73.2)$ & $56.5(52.3-60.7)$ & $68.6(64.9-72.5)$ \\
\hline & $4.5^{\mathrm{a}}$ & 85.1 (82.9-87.0) & $37.8(34.0-41.7)$ & $72.4(71.1-73.7)$ & $56.8(52.7-60.9)$ & $68.9(65.2-72.8)$ \\
\hline & 5 & $83.0(80.8-85.1)$ & $38.7(34.9-42.6)$ & $72.2(70.9-73.5)$ & $54.3(50.4-58.2)$ & $67.8(64.2-71.7)$ \\
\hline \multirow[t]{3}{*}{ ChPS } & 28 & $86.1(84.1-88.0)$ & $34.0(30.3-37.8)$ & $71.5(70.2-72.7)$ & $56.1(51.7-60.4)$ & $68.3(64.6-72.1)$ \\
\hline & 33 & $71.4(68.8-73.9)$ & $54.6(50.6-58.5)$ & 75.1 (73.3-76.8) & $49.9(47.0-52.7)$ & $65.6(62.0-69.4)$ \\
\hline & $38^{a}$ & $49.1(46.3-52.0)$ & $77.0(73.5-80.2)$ & $80.4(77.9-82.7)$ & $44.1(42.4-45.8)$ & $58.7(55.2-62.3)$ \\
\hline
\end{tabular}

Abbreviations: Cl confidence intervals, PPV Positive Predictive Value, NPV Negative Predictive Value, OA Overall Accuracy, PPI Palliative Prognostic Index, PS-PPI Performance Status-Based Palliative Prognostic Index; ChPS Chinese Prognostic Scale. ${ }^{a}$ Data were expressed as the best cutoff score based on Youden index (Youden index = sensitivity + specificity- 1 )

combination of initial PPI and score change was more accurate to predict the actual prognosis. Further studies are needed to modify the PPI for advanced cancer patients in the HPC setting.

In the previous study, [31] the PS-PPI was as accurate as the PPI to predict the survival of advanced cancer patients, which paralleled our findings that the AUROCs of the PPI and PS-PPI were not significantly different from each other. Survival time among the three risk groups of the PS-PPI was significantly different from each other. However, survival time between group B (4.1-6.0) and group C (6.1-15.0) in PPI was not significantly different from each other, which is not in accordance with previous findings $[30,50]$. One possible reason is that patients with PPS scores (30-50) accounted for $74.2 \%$ in the present study, which indicated that the performance status of patients might be overestimated by the physicians [10]. Another reason is that patients with moderate performance status could not be distinguished precisely by the KPS. The difference between the PPI and PS-PPI is that ECOG PS is used to take the place of PPS for performance status assessment. Performance status has been found to be strongly correlated with survival time in previous studies $[10,51-54]$. The European Association for Palliative Care has recommended the performance status as significant prognostic factors [1]. Myers 
et al. [55] reported that the ECOG scale, PPS, and KPS have a highly significant linear correlation. Another study reported by Chow et al. [56] suggested that there was a notable correlation of performance status scores among the ECOG scale, PPS, and KPS, and with no one tool statistically superior to others. In the present study, the KPS-to-ECOG and KPS-to-PPS conversion were based on the formula reported by Ma et al. [34] Thus, further studies are needed to compare the PPI and PSPPI for advanced cancer patients in the HPC setting.

Some other prognostic tools without blood test have been validated with good feasibility and accuracy in the HPC setting, such as the PiPS-A. The PiPS-A composed of thirteen factors was considered to be very useful and effective when laboratory results are unavailable [23]. Besides, Kim et al. also drew a similar conclusion [57]. However, the Karnofsky Performance Status (KPS) scores and the Quality of Life (QOL) scores are mainly used to assess the performance status of patients with advanced cancer in the HPC setting in mainland China, especially in the hospice center sponsored by the Li Ka Shing Foundation. Unfortunately, limited to the retrospective study, the global health status of patient, one of the basic prognostic parameters of the PiPS-A, could not be evaluated through the data provided by the hospice center. Hence, further studies are needed to validate the PiPS-A for patients with advanced cancer in the HPC setting in mainland China.

This study has some limitations. First, our study was carried out retrospectively and included only economically disadvantaged patients from a single institution, which may not be representative of patients with advanced cancer in the HPC setting in mainland China and worldwide. Second, ECOG PS scores and PPS scores were both transferred from KPS scores, which may affect the accuracy of the PPI and PS-PPI. Third, clinical characteristics of patients might be recorded in mistake without standardized specific assessment tools in a retrospective study. Notwithstanding these limitations, a large number of advanced cancer patients in the HPC setting were included in the present study. Meanwhile, the three prognostic tools we selected do not require blood tests and complicated calculations.

\section{Conclusions}

The present study demonstrated that the ChPS is more suitable than the PPI and PS-PPI for patients with advanced cancer in the HPC setting. More researches are needed to verify the predictive value of the ChPS, PPI, and PS-PPI in HPC settings in the future.

\section{Abbreviations}

HPC: Home-Based Palliative Care; PPI: Palliative Prognostic Index; PSPPI: Performance Status-Based Palliative Prognostic Index; ChPS: Chinese Prognosis Scale; ECOG: Eastern Cooperative Oncology Group; PPS: Palliative
Performance Scale; KPS: Karnofsky Performance Status; QOL: Quality of Life; AUROC: The Area Under the Receiver Operating Characteristic Curve; PPV: Positive Predictive Value; NPV: Negative Predictive Value; Cl: Confidence Intervals; SD: Standard Deviation

\section{Acknowledgements}

We are grateful to the Hospice Center of Yuebei People's Hospital Affiliated to Shantou University Medical College for their assistance in the use of case data.

\section{Authors' contributions}

The study was designed by QW, XHD, YZB, YZH and FJL. The data were collected and analysed by JZ, STX, ZYC, JT, XF, LQ, FPZ, MCH, YW and XRZ. The results of the analysis were discussed and approved by all authors. JZ, STX and XHD drafted the manuscript. QW, YZB, YZH and FJ reviewed the manuscript. All authors read and approved the final manuscript. JZ and STX contributed equally to this work.

\section{Funding}

This work was supported by the Medical and Health Scientific Research Project of Science and Technology Bureau of Shaoguan City (2019sn003). The funder has no influence on study design; collection, management, analysis, and interpretation of data; writing the report; and the decision to submit the report for publication.

\section{Availability of data and materials}

The datasets used and/or analysed during the current study are available from the corresponding author on reasonable request.

\section{Ethics approval and consent to participate}

The study was approved by the ethical review board of Yuebei People's Hospital Affiliated to Shantou University Medical College (KY-2019-024) and was performed according to the ethical standards laid down in the 1964 Declaration of Helsinki and its later amendments or comparable ethical standards. Written informed consent was obtained from all individual or guardian participants.

\section{Consent for publication}

Not Applicable.

\section{Competing interests}

The authors declare that they have no competing interests.

\section{Author details}

${ }^{1}$ Department of Spine Surgery, Yuebei People's Hospital Affiliated to Shantou University Medical College, Shaoguan, Guangdong, China. ${ }^{2}$ Department of Nursing, Yuebei People's Hospital Affiliated to Shantou University Medical College, Shaoguan, Guangdong, China. ${ }^{3}$ Hospice center of Yuebei People's Hospital Affiliated to Shantou University Medical College, Shaoguan,

Guangdong, China. ${ }^{4}$ Emergency rescue command center of Shaoguan city, Shaoguan, Guangdong, China. ${ }^{5}$ Hospice center of Fourth Affiliated Hospital of Guangxi Medical University, Liuzhou, Guangxi, China. ${ }^{6}$ Department of Spinal Surgery, Nanfang Hospital, Southern Medical University, Guangzhou, Guangdong, China.

Received: 17 May 2020 Accepted: 22 October 2020

Published online: 31 October 2020

\section{References}

1. Maltoni M, Caraceni A, Brunelli C, Broeckaert B, Christakis N, Eychmueller S, et al. Prognostic factors in advanced cancer patients: evidence-based clinical recommendations - A study by the steering Committee of the European Association for palliative care. J Clin Oncol. 2005;23(25):6240-8.

2. Glare PA, Sinclair CT. Palliative medicine review: prognostication. J Palliat Med. 2008;11(1):84-103.

3. Finlay E, Casarett D. Making difficult discussions easier: using prognosis to facilitate transitions to hospice. CA Cancer J Clin. 2009;59(4):250-63.

4. Stone PC, Lund S. Predicting prognosis in patients with advanced cancer. Ann Oncol. 2007;18(6):971-6. 
5. Yoong J, Atkin N, Le B. Use of the palliative prognostic index in a palliative care consultation service in Melbourne, Australia. J Pain Symptom Manage. 2010;39(1):e2-4.

6. Glare P, Virik K, Jones M, Hudson M, Eychmuller S, Simes J, et al. A systematic review of physicians' survival predictions in terminally ill cancer patients. BMJ. 2003;327(7408):195.

7. Christakis NA, Lamont EB. Extent and determinants of error in doctors' prognoses in terminally ill patients: prospective cohort study. BMJ. 2000; 320(7233):469-73.

8. Gripp S, Moeller S, Bölke E, Schmitt G, Matuschek C, Asgari S, et al. Survival prediction in terminally ill cancer patients by clinical estimates, laboratory tests, and self-rated anxiety and depression. J Clin Oncol. 2007;25(22):3313-20.

9. Morita T, Tsunoda J, Inoue S, Chihara S. Improved accuracy of physicians survival prediction for terminally ill cancer patients using the palliative prognostic index. Palliat Med. 2001;15(5):419-24.

10. Lingjun $Z$, Jing $C$, Jian $L$, Wee $B$, Jijun $Z$. Prediction of survival time in advanced Cancer: A prognostic scale for Chinese patients. J Pain Symptom Manag. 2009;38(4):578-86.

11. Ning XH. Hospice and Palliative Care in Mainland China: History, Current Status and Challenges. Chin Med Sci J. 2018; doi: https://doi.org/10.24920/ 003524.

12. Zheng R, Sun K, Zhang S, Zeng H, Zou X, Chen R, et al. Report of cancer epidemiology in China, 2015. Chin J Oncol. 2019. https://doi.org/10.3760/ cma.j.jssn.0253-3766.2019.01.005.

13. Deng D, Lin W, Law F. The study on evaluation and improvement of quality of life in patients with advanced Cancer by China's hospice program. Am J Hosp Palliat Me. 2014;32(4):365-71.

14. Simmons CPL, McMillan DC, McWilliams K, Sande TA, Fearon KC, Tuck S, et al. Prognostic tools in patients with advanced Cancer: A systematic review. J Pain Symptom Manag. 2017. https://doi.org/10.1016/j. jpainsymman.2016.12.330.

15. Pirovano $M$, Maltoni $M$, Nanni $O$, Marinari M, Indelli M, Zaninetta $G$, et al. A new palliative prognostic score: a first step for the staging of terminally ill cancer patients. Italian multicenter and study group on palliative care. J Pain Symptom Manag. 1999;17(4):231-9.

16. Scarpi E, Maltoni M, Miceli R, Mariani L, Caraceni A, Amadori D, et al. Survival prediction for terminally ill cancer patients: revision of the palliative prognostic score with incorporation of delirium. Oncologist. 2011;16(12):1793-9.

17. Glare PA, Eychmueller S, McMahon P. Diagnostic accuracy of the palliative prognostic score in hospitalized patients with advanced cancer. J Clin Oncol. 2004;22(23):4823-8.

18. Tarumi Y, Watanabe SM, Lau F, Yang J, Quan H, Sawchuk L, et al. Evaluation of the palliative prognostic score (PaP) and routinely collected clinical data in prognostication of survival for patients referred to a palliative care consultation service in an acute care hospital. J Pain Symptom Manag. 2011; 42(3):419-31.

19. Numico G, Occelli M, Russi EG, Silvestris N, Pasero R, Fea E, et al. Survival prediction and frequency of anticancer treatment in cancer patients hospitalized due to acute conditions. Role of clinical parameters and PaP score. Support Care Cancer. 2011;19(11):1823-30

20. Glare P, Virik K. Independent prospective validation of the PaP score in terminally ill patients referred to a hospital-based palliative medicine consultation service. J Pain Symptom Manag. 2001;22(5):891-8.

21. Anderson F, Downing GM, Hill J, Casorso L, Lerch N. Palliative performance scale (PPS): A new tool. J Palliat Care. 1996;12(1):5-11.

22. Hyodo I, Morita T, Adachi I, Shima Y, Yoshizawa A, Hiraga K. Development of a predicting tool for survival of terminally ill Cancer patients. Jpn J Clin Oncol. 2010;40(5):442-8.

23. Baba M, Maeda I, Morita $T$, Inoue S, Ikenaga M, Matsumoto $Y$, et al. Survival prediction for advanced cancer patients in the real world: A comparison of the palliative prognostic score, delirium-palliative prognostic score, palliative prognostic index and modified prognosis in palliative care study predictor model. Eur J Cancer. 2015;51(12):1618-29.

24. Morita T, Tsunoda J, Tsunoda J, Chihara S. The palliative prognostic index: a scoring system for survival prediction of terminally ill cancer patients. Support Care Cancer. 1999;7(3):128-33.

25. Stone CA, Tiernan E, Dooley BA. Prospective validation of the palliative prognostic index in patients with cancer. J Pain Symptom Manag. 2008; 35(6):617-22.

26. Sonoda H, Yamaguchi T, Matsumoto M, Hisahara K. Validation of the palliative prognostic index and palliative prognostic score in a palliative care consultation team setting for patients with advanced cancers in an acute care hospital in Japan. Am J Hosp Palliat Me. 2014;31(7):730-4.

27. Hamano J, Maeno T, Kizawa Y, Shima Y, Maeno T. Usefulness of palliative prognostic index for patient with advanced cancer in home care setting. Am J Hosp Palliat Me. 2013;30(3):264-7.

28. Alshemmari S, Ezzat H, Samir Z, Refaat S, Alsirafy SA. The palliative prognostic index for the prediction of survival and in-hospital mortality of patients with advanced cancer in Kuwait. J Palliat Med. 2012;15(2):200-4.

29. Hamano J, Kizawa Y, Maeno T, Nagaoka H, Shima Y, Maeno T. Prospective clarification of the utility of the palliative prognostic index for patients with advanced cancer in the home care setting. Am J Hosp Palliat Me. 2014; 31(8):820-4

30. Maltoni M, Scarpi E, Pittureri C, Martini F, Montanari L, Amaducci E, et al. Prospective comparison of prognostic scores in palliative care Cancer populations. Oncologist. 2012:17(3):446-54.

31. Yamada T, Morita T, Maeda I, Inoue S, Ikenaga M, Matsumoto Y, et al. A prospective, multicenter cohort study to validate a simple performance statusbased survival prediction system for oncologists. Cancer. 2017;123(8):1442-52.

32. $\mathrm{KD} \mathrm{A}, \mathrm{BJ} \mathrm{H}$. The clinical evaluation of chemotherapeutic agents in cancer. In: MacLeod C, editor. Evaluation of chemotherapeutic agents. New York: Columbia University Press; 1949. p. 196.

33. Sun Y. China: status of cancer pain and palliative care. J Pain Symptom Manag. 1993:8(6):399-403.

34. Ma C, Bandukwala S, Burman D, Bryson J, Seccareccia D, Banerjee S, et al. Interconversion of three measures of performance status: an empirical analysis. Eur J Cancer. 2010;46(18):3175-83.

35. Oken MM, Creech RH, Tormey DC, Horton J, Davis TE, McFadden ET, et al. Toxicity and response criteria of the eastern cooperative oncology group. Am J Clin Oncol. 1982;5(6):649-55.

36. DeLong ER, DeLong DM, Clarke-Pearson DL. Comparing the areas under two or more correlated receiver operating characteristic curves: a nonparametric approach. Biometrics. 1988:44(3):837-45.

37. Yoon S-J, Jung J-G, Kim J-S, Kim S-S, Kim S. Retrospective assessment of objective prognostic score in terminally ill Korean patients with cancer. Am J Hosp Palliat Me. 2014;31(4):435-40.

38. Yoon SJ, Suh SY, Lee YJ, Park J, Hwang S, Lee SS, et al. Prospective validation of objective prognostic score for advanced Cancer inpatients in South Korea: A multicenter study. J Palliat Med. 2017:20(1):65-8.

39. Katagiri H, Okada R, Takagi T, Takahashi M, Murata H, Harada H, et al. New prognostic factors and scoring system for patients with skeletal metastasis. Cancer Med. 2014;3(5):1359-67.

40. Lei M, Li J, Liu Y, Jiang W, Liu S, Zhou S. Who are the best candidates for Decompressive surgery and spine stabilization in patients with metastatic spinal cord compression?: A new scoring system. Spine. 2016;41(18):1469-76.

41. Kobayashi K, Ando K, Nakashima H, Sato K, Kanemura T, Yoshihara H, et al. Prognostic Factors in the New Katagiri Scoring System after Palliative Surgery for Spinal Metastasis. Spine. 2020.

42. Yin Z, Tang H, Li L, Ni J, Yuan S, Lou H, et al. Impact of sites versus number of metastases on survival of patients with organ metastasis from newly diagnosed cervical cancer. Cancer Manag Res. 2019;11:7759-66.

43. Abdel-Rahman O. Clinical correlates and prognostic value of different metastatic sites in metastatic renal cell carcinoma. Future Oncol. 2017; 13(22):1967-80.

44. Deng Y, Bi R, Zhu Z, Li S, Xu B, Rather WA, et al. A surveillance, epidemiology and end results database analysis of the prognostic value of organ-specific metastases in patients with advanced prostatic adenocarcinoma. Oncol Lett. 2019;18(2):1057-70.

45. Jho HJ, Suh SY, Yoon SJ, Lee SS, Ahn HY, Yamaguchi T, et al. Prospective validation of the objective prognostic score for advanced Cancer patients in diverse palliative settings. J Pain Symptom Manag. 2016;52(3):420-7.

46. Yoon S-J, Jung J-G, Kim J-S, Kim S-S, Kim S. Comparison of accuracy among prognostic scores for predicting life expectancy in Korean patients with cancer with weeks of survival. Am J Hosp Palliat Me. 2014;31(8):845-52.

47. Subramaniam S, Dand P, Ridout M, Cawley D, Miller S, Valli P, et al. Prognosis prediction with two calculations of palliative prognostic index: further prospective validation in hospice cancer patients with multicentre study. BMJ Support Palliat Care. 2019;9(3):326-31.

48. Kao C-Y, Hung Y-S, Wang H-M, Chen J-S, Chin T-L, Lu C-Y, et al. Combination of initial palliative prognostic index and score change provides a better prognostic value for terminally ill Cancer patients: A sixyear observational cohort study. J Pain Symptom Manag. 2014;48(5):804-14. 
49. Arai Y, Okajima Y, Kotani K, Tamba K. Prognostication based on the change in the palliative prognostic index for patients with terminal Cancer. J Pain Symptom Manag. 2014;47(4):742-7.

50. Stiel S, Bertram L, Neuhaus S, Nauck F, Ostgathe C, Elsner F, et al. Evaluation and comparison of two prognostic scores and the physicians' estimate of survival in terminally ill patients. Support Care Cancer. 2010;18(1):43-9.

51. Chow E, Harth T, Hruby G, Finkelstein J, Wu J, Danjoux C. How accurate are physicians' clinical predictions of survival and the available prognostic tools in estimating survival times in terminally ill cancer patients? A systematic review. Clin Oncol. 2001;13(3):209-18.

52. Chow E, Abdolell M, Panzarella T, Harris K, Bezjak A, Warde P, et al. Predictive model for survival in patients with advanced cancer. J Clin Oncol. 2008;26(36):5863-9.

53. Glare $P$, Sinclair C, Downing M, Stone $P$, Maltoni M, Vigano A. Predicting survival in patients with advanced disease. Eur J Cancer. 2008:44(8):1146-56.

54. Dajczman E, Kasymjanova G, Kreisman H, Swinton N, Pepe C, Small D. Should patient-rated performance status affect treatment decisions in advanced lung cancer? J Thorac Oncol. 2008;3(10):1133-6.

55. Myers J, Gardiner K, Harris K, Lilien T, Bennett M, Chow E, et al. Evaluating correlation and interrater reliability for four performance scales in the palliative care setting. J Pain Symptom Manag. 2010;39(2):250-8.

56. Chow R, Bruera E, Temel JS, Krishnan M, Im J, Lock M. Inter-rater reliability in performance status assessment among healthcare professionals: an updated systematic review and meta-analysis. Support Care Cancer. 2020.

57. Kim ES, Lee JK, Kim MH, Noh HM, Jin YH. Validation of the prognosis in palliative care study predictor models in terminal cancer patients. Korean J Fam Med. 2014;35(6):283-94.

\section{Publisher's Note}

Springer Nature remains neutral with regard to jurisdictional claims in published maps and institutional affiliations.

Ready to submit your research? Choose BMC and benefit from:

- fast, convenient online submission

- thorough peer review by experienced researchers in your field

- rapid publication on acceptance

- support for research data, including large and complex data types

- gold Open Access which fosters wider collaboration and increased citations

- maximum visibility for your research: over $100 \mathrm{M}$ website views per year

At $\mathrm{BMC}$, research is always in progress.

Learn more biomedcentral.com/submissions 